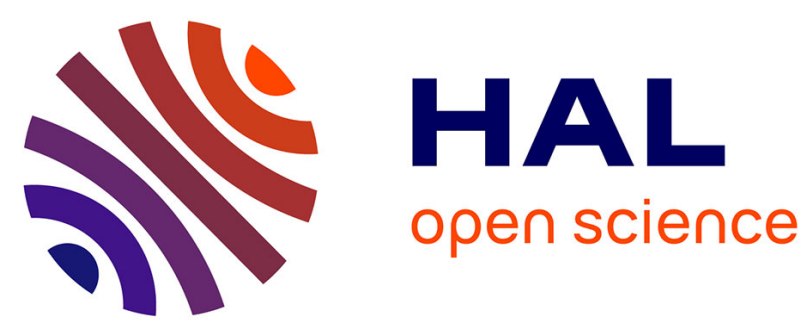

\title{
Antiviral activity of Formyl Peptide Receptor 2 antagonists against influenza viruses
}

Noemie Courtin, Aurelien Fotso Fotso, Pierre Fautrad, Floriane Mas, Marie-Christine Alessi, Beatrice Riteau

\section{- To cite this version:}

Noemie Courtin, Aurelien Fotso Fotso, Pierre Fautrad, Floriane Mas, Marie-Christine Alessi, et al.. Antiviral activity of Formyl Peptide Receptor 2 antagonists against influenza viruses. Antiviral Research, 2017, 143, pp.252-261. 10.1016/j.antiviral.2017.05.001 . hal-01755130

\section{HAL Id: hal-01755130 \\ https://hal-amu.archives-ouvertes.fr/hal-01755130}

Submitted on 30 Mar 2018

HAL is a multi-disciplinary open access archive for the deposit and dissemination of scientific research documents, whether they are published or not. The documents may come from teaching and research institutions in France or abroad, or from public or private research centers.
L'archive ouverte pluridisciplinaire HAL, est destinée au dépôt et à la diffusion de documents scientifiques de niveau recherche, publiés ou non, émanant des établissements d'enseignement et de recherche français ou étrangers, des laboratoires publics ou privés. 
Antiviral activity of Formyl Peptide Receptor 2 antagonists against influenza viruses

Noémie Courtin, Aurélien Fotso Fotso, Pierre Fautrad, Floriane Mas, Marie-Christine Alessi and Béatrice Riteau

Institut National de la Santé et de la Recherche Médicale (Inserm), UMR_S 1062, 13005 Marseille, France Inra, UMR_INRA 1260, 13005 Marseille, France Aix Marseille Université, 13005 Marseille, France.

correspondance to Beatrice Riteau: beatrice.riteau@univ-amu.fr 


\begin{abstract}
Influenza viruses are one of the most important respiratory pathogens worldwide, causing both epidemic and pandemic infections. The aim of the study was to evaluate the effect of FPR2 antagonists PBP10 and BOC2 on influenza virus replication. We determined that these molecules exhibit antiviral effects against influenza A (H1N1, H3N2, H6N2) and B viruses. FPR2 antagonists used in combination with oseltamivir showed additive antiviral effects. Mechanistically, the antiviral effect of PBP10 and BOC2 is mediated through early inhibition of virus-induced ERK activation. Finally, our preclinical studies showed that FPR2 antagonists protected mice from lethal infections induced by influenza, both in a prophylactic and therapeutic manner. Thus, FPR2 antagonists might be explored for novel treatments against influenza.
\end{abstract}

Keywords: Formyl Peptide Receptor 2, influenza virus, antiviral 


\section{INTRODUCTION}

Influenza is a major public health problem (Kuiken et al., 2012). Annually, influenza epidemics cause 200-500,000 fatal cases worldwide. Viruses of animal origin (mainly avian) can occasionally be transmitted to humans and become pandemic, whose impact can range from mild to severe (40 million deaths for the Spanish Flu). The etiological agents of the disease, the single-stranded RNA influenza viruses, are classified into four types (A, B, C and D). Both influenza A (IAV) and B viruses are responsible for epidemics. IAV are further divided into subtypes based on their surface glycoproteins, hemagglutinin (HA, 18 subtypes) and neuraminidase (NA, 11 subtypes).

Current antiviral drugs used for these pathogens are limited to two approved classes of compounds that target viral proteins, thereby promoting selection pressure. The adamantane compounds block the viral M2 ion channel protein, whereas oseltamivir and zanamivir bind the viral enzyme neuraminidase (NA). Particularly regarding adamantanes, a drastic increase in viral resistance has occurred in recent years (Hayden and de Jong, 2011). The substitution of a single amino acid can make a mutant virus resistant without affecting its virulence (Hayden and de Jong, 2011). This illustrates the urgent need for novel antiviral approaches. In this manuscript, to overcome this challenge of resistance, a cellular molecule was targeted instead of the virus.

The Formyl Peptide Receptors (FPRs) belong to the G protein-coupled receptors (Le et al., 2002), in which three FPRs with similarities in their amino acid sequences, were described in humans (FPR1, 2 and 3). FPR2 also known as FPRL1 (Formyl Peptide receptor-like 1) or ALX (lipoxin A4 receptor) binds different kinds of ligands: formyl peptides, whose major biological source is bacteria, fatty acid lipid mediators [such as lipoxin A4 (LXA4)] and 
cellular proteins (such as Annexin-1). FPRs might be important receptors in viral pathogenesis. Indeed, FPR1 is activated by the nonstructural protein $5 \mathrm{~A}$ of hepatitis $\mathrm{C}$ viruses inducing activation and migration of human phagocytes (Lin et al., 2011). Regarding FPR2, it is used by immunodeficiency viruses (IV) as a co-receptor for viral replication (Nedellec et al., 2009) both for human IV-1 isolates (Shimizu et al., 2008a) and simian IV (Shimizu et al., 2008b). In addition, the gp41 and gp120 of HIV-1 activate FPR2 on human phagocytes and monocytes, leading to activation and desensitization of cell immune response, respectively (Deng et al., 1999; Su et al., 1999). More recently, we found that FPR2 has a proviral role during IAV infections and increases virus pathogenesis (Tcherniuk et al., 2016). Inhibiting FPR2-signaling with the FPR2 antagonist WRW4 inhibited IAV replication and protected mice from lethal IAV infections. Altogether, these reports illustrate how FPR2 can be used by several viruses to support their own replication.

The aim of this study was to go further into the identification of molecules targeting FPR2 in order to foster the development of FPR2 antagonists as antiviral molecules against influenza. The present report shows that the FPR2 antagonists PBP10 and BOC2 (Ortiz-Munoz et al., 2014; Skovbakke et al., 2015), are two novel potent antiviral inhibitors of both influenza A and B viruses. Thus, FPR2 is a potential tractable target for treating a broad range of influenza viruses. 


\section{MATERIALS AND METHODS}

\subsection{Viruses, cells and reagents}

IAV A/PR/8/34 (H1N1), A/HK/68 (H3N2) and influenza B virus B/70 were a gift from GF. Rimmelzwaan (Erasmus Medical Center, Rotterdam, the Netherlands). A/Turkey/Massachussets/65 (H6N2) was a gift from V. Jestin (Agence nationale de sécurité sanitaire de l'alimentation, de l'environnement et du travail, Maisons-alfort, France). The human alveolar A549 and the Madin-Darby canine kidney (MDCK) cell lines used in this study were a gift from GF. Rimmelzwaan. Cells were cultured as previously described (Berri et al., 2014). The following reagents were used in the study: monoclonal anti-tubulin antibody (Sigma Aldrich), polyclonal anti-p-ERK antibody (Cell Signaling Technology), anti-viral M2 protein (Santa Cruz), and oseltamivir (Sigma-Aldrich). The ERK inhibitor pathway U0126 was obtained from Sigma-Aldrich.

WRW4 (Trp-Arg-Trp-Trp-Trp-Trp- $\mathrm{NH}_{2}$ ) is a selective antagonist of FPR2. It inhibits the binding of WKYMVm (FPR2 agonist) to FPR2, resulting in the complete inhibition of ERK signalling as well as intracellular calcium increase (Bae et al., 2004). WRW4 was obtained from Alomone Labs. PBP10 (RhoB-Glu-Arg-Leu-Phe-Glc-Val-Lys-Glc-Arg-Arg) is a 10-aalong rhodamine-linked and membrane-permeable peptide inhibitor. It adopts a phosphatidylinositol 4,5-bisphosphate-binding sequence in the cytoskeletal protein gelsolin. Its activity might depend on its ability to pass membrane, disassemble actin filament structures and FPR2-mediated cellular response (Cunningham et al., 2001). It is highly specific to FPR2 and has no inhibitory function on FPR1. PBP10 was obtained from Tocris. BOC2 (Boc-Phe-Leu-Phe-Leu-Phe-OH ) is a competitive antagonist of the binding of formyl peptides to FPR. BOC2 blocks both human and murine FPR2 (Verriere et al., 2012; Vital et al., 2016). It also inhibits FPR1 signalling. BOC2 was obtained from CliniSciences. 


\subsection{Infection experiments and cell viability}

A549 cells were pre-incubated for 20 minutes with or without the indicated concentration of FPR2 antagonist, WRW4, PBP10 or BOC2 before being infected with the indicated influenza virus (MOI 1). In some experiments, assays were performed in the presence of $0.25 \mu \mathrm{M}$ U0126 or vehicle. After one hour, the virus was removed and medium containing the abovementioned FPR2 inhibitors was added in the presence or absence of the indicated concentrations of oseltamivir to let virus replication proceed. At the indicated time point postinfection, infectious virus titers were assessed in the supernatant or RNA was extracted. Cell viability in the presence of FPR2 antagonists was assessed by trypan blue staining 72 hours post-treatment.

\subsection{Real-Time quantitative PCR analyses}

Total RNA was extracted for each experimental condition from A549 cells using QIAzol reagent (Qiagen) according to the manufacturer's protocol. $5 \mu \mathrm{g}$ of the resulting RNA was then reverse transcribed using the M-MLV Reverse Transcriptase kit (Invitrogen). Regarding vRNA (viral RNA) reverse transcription, Uni12 primer was used as previously described (Hoffmann et al., 2001). A specific primer for GAPDH was used for reverse transcription (Baier et al., 1993) as housekeeping gene. Real-Time qPCRs were then performed with the 5X HOT Pol EvaGreen qPCR Mix Plus (Invitrogen). Amplification plots were generated using the LightCycler 480 software (Roche), and fold induction was calculated using the threshold cycle method $\left(2^{-\Delta \Delta C t}\right)$, GAPDH was used for normalization.

Primer sequences were the following:

Gene

Primer sequence ${ }^{\mathrm{a}}$ 


\begin{tabular}{|l|l|}
\hline Viral NS1 & Fw: 5'-CTGTGTCAAGCTTTCAGGTAGA- \\
& $3 '$ \\
\hline Human GADPH & Rv: 5'-GGTACAGAGGCCATGGTCAT-3' \\
\hline Uni12 & Fw: 5'-GAAGGTGAAGGTCGGAGT-3' \\
\hline GAPDH RT & Rv' 5'-GAAGATGGTGATGGGATTTC-3' \\
\hline a Fw, forward; Rv, reverse & 5'-GAGATGATGACCCTTTTGGC-3' \\
\hline
\end{tabular}

\subsection{Virus production and titration}

Virus production was performed on MDCK cells that were seeded at $15 \times 10^{6}$ cells per 75 $\mathrm{cm} 2$ tissue culture flask and incubated at $37^{\circ} \mathrm{C}$ overnight. The next day, cells were infected with IAV at a multiplicity of infection (MOI) of $10-3$ in medium containing $1 \mu \mathrm{g} / \mathrm{ml}$ of trypsin. Two days post-infection, the supernatant was harvested, purified by centrifugation and subsequently the virus particles were frozen at $-80^{\circ} \mathrm{C}$. For viral titration, MDCK cells were grown in 6 well culture plates and infected with serial dilutions of the supernatant containing the infectious viruses for one hour, at $37^{\circ} \mathrm{C}$. After adsorption, cells were overlaid with medium containing $2 \%$ agarose and $1 \mu \mathrm{g} / \mathrm{ml}$ of trypsin and incubated for 3 days, at $37^{\circ} \mathrm{C}$. Viral plaques were then visualized by crystal violet staining.

\subsection{Fluorescence Microscopy Experiments}

Fluorescence microscopy was performed as previously described (Berri et al., 2014). Briefly, A549 cells were seeded and cultured on glass coverslips in a multiwell plate. The next day, infection experiments in the presence or absence of PBP10 or BOC2 were performed as 
described above. Cells were then fixed and permeabilized with $4 \%$ paraformaldehyde containing $0,2 \%$ Triton-X100. Cells were then extensively washed with phosphate-buffered saline and were incubated with a viral anti-M2 primary antibody for 1 hour at $37^{\circ} \mathrm{C}$. Subsequently, an Alexa Fluor (Life Technologies) secondary antibody was used for 1 hour at $37^{\circ} \mathrm{C}$. Cells were counterstained with DAPI for 15 minutes. Images were analyzed using a Zeiss IMAGER.M1.

\subsection{ERK activation experiments}

Regarding the kinetic of virus-induced ERK phosphorylation, A549 cells were incubated with IAV A/PR/8/34 (MOI 10) at the indicated time point before cell lysis. Regarding the effect of PBP10 or BOC2, A549 cells were first pretreated for 20 minutes at $37^{\circ} \mathrm{C}$ with FPR2 antagonists PBP10 or BOC2. Cells were then incubated with A/PR/8/34 virus (MOI 10) for 5 minutes and then lysed in ice-cold lysis buffer (1\% Triton X-100, $100 \mathrm{mM}$ Tris-HCl [pH 7.4], 1.5 $\mathrm{M} \mathrm{NaCl}, 5 \mathrm{mM}$ EDTA, in the presence of a complete proteinase inhibitor mixture). Proteins from the lysates were then analyzed by western blot, as previously described (Riteau et al., 2003).

\subsection{In vivo experiments}

Five- to six-week-old female C57BL/6 mice (Charles River) were anesthetized with Ketamine/Xylazine $(43 / 5 \mathrm{mg} / \mathrm{kg})$ and inoculated intranasally with $20 \mu \mathrm{l}$ of a solution containing A/PR/8/34 virus, as previously described(Berri et al., 2013; Le et al., 2015). Regarding BOC2 treatment $(4 \mathrm{mg} / \mathrm{kg})$, mice were treated once the day of virus inoculation (500 PFU). Prophylactic treatment with WRW4 $(8 \mathrm{mg} / \mathrm{kg})$ or BOC2 $(4 \mathrm{mg} / \mathrm{kg})$ was achieved by treating mice once, one day before virus inoculation (750 PFU). WRW4 and BOC2 were both administered intraperitoneally. Upon virus inoculation, survival rates and loss of body 
weight was scored daily. At the end of the experiment, mice were sacrificed by cervical dislocation. For assessing virus replication, BAL was harvested from sacrificed mice, and infectious virus titers were determined by plaque assay. Total protein was evaluated by using the Coomassie Bradford Protein assay kit (Thermo Scientific, Franklin, MA). The protocol was approved by the committee of animal experiments of the Faculty of Marseille la Timone (number: G130555). All animal experiments were also carried out under the authority of a license issued by "la direction des services Vétérinaires" (accreditation 693881479). Twenty percent weight loss was used as end point for the mortality rate.

\subsection{Statistical analysis}

All statistical analyses were performed using GraphPad Prism software. The Mann-Whitney test was used for statistical analysis, regarding viral replication. Results were analysed using a Wilcoxon test or one-way analysis of variance (ANOVA) for real-time PCR analysis. Differences in survival rates were analyzed using a Log-Rank (Mantel-Cox) test. Results were considered statistically significant at $p<0.05(*), p<0.01(* *), P<0,0001(* * * *)$. 


\section{RESULTS}

\subsection{Treatment of $A 549$ cells with $\mathrm{PBP10}$ or BOC2 inhibits $\mathrm{A} / \mathrm{PR} / 8 / 34$ virus replication}

To examine the antiviral properties of PBP10 and BOC2 against IAV, we first evaluated the cytotoxic effects of A549 cell treatment with different concentrations of PBP10 or BOC2 (1.25-320 $\mu \mathrm{M})$. After an incubation period of 72 hours, we observed that $10 \mu \mathrm{M}-320 \mu \mathrm{M}$ of PBP10 and $320 \mu \mathrm{M}$ of BOC2 were cytotoxic to the cells, as measured by blue trypan staining (Figure1A). We thus determined that $5 \mu \mathrm{M}$ will be used in the subsequent experiments for both PBP10 and BOC2, a concentration also used by others (Fu et al., 2004).

Then, to investigate whether PB10 and BOC2 treatment would affect the release of infectious viral particles, A549 cells were infected with A/PR/8/34 virus and pretreated or not with the indicated dose of FPR2 antagonist PBP10 or BOC2. At different time points post-infection, infectious viral titers were then assessed by classical plaque assays. Treatment of IAVinfected cells with FPR2 antagonist significantly reduced viral production in a time course and dose-dependent manner (Figure1 B-C).

To confirm and visualize the antiviral effect of PBP10 and BOC2, immunofluorescence staining was performed. A549 cells were pretreated with each inhibitor and infected with IAV for 24 hours. The expression of the viral M2 protein was then assessed by immunofluorescence microscopy, using a specific anti-M2 antibody. Results showed that in untreated infected cells, M2 was highly expressed and distributed in the cytoplasm and at the cell membrane of all infected cells (Figure 2A). In contrast, in the presence of PBP10 or BOC2, not only the intensity of fluorescence was reduced but also a large percentage of cells showed no significant M2 expression. In these assays, nuclei were stained with DAPI and the merged panels are shown. As controls, uninfected cells displayed undetectable M2 proteins 
(lower panels). Altogether, these results indicated that cell treatment with PBP10 and BOC2 leads to decreased $\mathrm{A} / \mathrm{PR} / 8 / 34$ virus production in infected cells.

To determine the step in viral replication that the drugs are blocking, we evaluated the mRNA and vRNA levels of the viral NS1 (nonstructural 1) protein in A/PR/8/34 (H1N1) infected A549 alveolar epithelial cells, pretreated or not with $5 \mu \mathrm{M}$ of PBP10 or BOC2. Results showed that compared to infected untreated cells, cell pretreatment with PBP10 or BOC2 significantly reduced the NS1 mRNA and vRNA expression level in infected cells (Figure 2B). Thus, these results show that BOC2 and PBP10 inhibit viral genome replication.

\subsection{PBP10 and BOC2 inhibits $\mathrm{A} / \mathrm{PR} / 8 / 34$ virus replication through $\mathrm{ERK}$ activation}

We previously reported that IAV promoted its own replication through binding to FPR2. Subsequently, this leads to ERK activation, a signaling pathway required for virus life cycle (Tcherniuk et al., 2016). Thus, we next tested whether the antiviral effect of PBP10 and BOC2 occurred through blocking influenza-virus induced ERK activation. First, we confirmed that binding of A/PR/8/34 virus to A549 cells promoted ERK phosphorylation after 5 minutes (Figure 3A-B, left panels). This phosphorylation remained elevated after 10 or 30 minutes and decreased after 60 minutes. More importantly, A549 cell pre-treatment with FPR2 antagonist PBP10 or BOC2 prevented IAV-induced ERK activation after 5 minutes binding but not thereafter (Figure 3A-B, right panels). Thus, PBP10 and BOC2 delayed IAVFPR2 recognition, leading to impaired early ERK activation. To evaluate the role of this signaling pathway in the antiviral activity of PBP10 and BOC2, A549 cells were pre-treated with either FPR2 antagonist in the presence or absence of the ERK pathway inhibitor, U0126. Afterwards, infectious virus titers were evaluated by plaque assays. In absence of U0126, cell treatment with PBP10 alone decreased virus production by A/PR/8/34 virus-infected cells (Figures 3C, left panel). As expected, cell treatment with U0126 alone also showed antiviral 
activity. However, in the presence of U0126, the difference in viral replication between untreated and PBP10-treated cells was abrogated. Similar results were also observed using BOC2 to inhibit FPR2 (Figure 3C, right panel). Thus, PBP10 and BOC2 blocked viral replication through FPR2 induced-ERK activation.

\subsection{Combined treatment of FPR2 antagonists with Oseltamivir}

We next assessed the antiviral efficacy of combined treatment of FPR2 antagonists with oseltamivir. Infectious virus titers in the supernatant of IAV-infected A549 cells treated or not with PBP10, oseltamivir or a combination of PBP10 and oseltamivir were evaluated. As expected, cell treatment with either oseltamivir or PBP10 alone showed antiviral activity against A/PR/8/34 virus (Figure 4A, left panel). The effect of co-treatment of PBP10 with oseltamivir on inhibition of viral replication was additive. Similar results were observed when BOC2 was used as the FPR2 antagonist (Figure 4A, right panel). Thus, FPR2 antagonist used in combination with oseltamivir boosts the antiviral activity.

\subsection{Antiviral effect of FPR2 antagonists on several influenza A virus strains and B viruses}

The antiviral activity of the FPR2 antagonists was then evaluated against other subtypes of IAV strains as well as influenza B viruses. A549 cells were left untreated or were pre-treated with either PBP10, BOC2 or WRW4 $(5 \mu \mathrm{M})$. Cells were then infected with B/NL/076/06 influenza B viruses, A/HK/68 IAV (H3N2) or A/Turkey/Massachusetts/65. After 24 hours post-infection, the supernatant was collected and infectious virus titers were analyzed by classical plaque assays. Results showed that all viruses replicated efficiently in untreated cells. However, cell treatment with WRW4, PBP10 or BOC2 (Figure 5A-C) significantly 
inhibited virus replication. Thus, we concluded that FPR2 antagonists have an antiviral effect on different influenza viruses.

\subsection{Protective effect of FPR2 antagonists in vivo}

Our previous report showed that mice treatment with WRW4 protected them from IAVinduced death (Tcherniuk et al., 2016). Thus, we next investigated whether another antagonist of FPR2 would have the same effect. Results showed that mice treated with BOC2 were significantly more resistant to A/PR/8/34 infection than vehicle-treated mice (Figure 6A). In contrast, treatment of uninfected mice with BOC2 did not affect their survival rates (not shown), which suggests that FPR2 antagonists do not cause side effects. Finally, we investigated whether blocking FPR2 in a prophylactic manner was still protective. When BOC2 or WRW4 FPR2 antagonists were administered once and one day before inoculation, mice were also significantly protected from A/PR/8/34 virus infections (Figure 6B-C). In addition, virus replication and total proteins in BAL of WRW4-treated mice were significantly decreased compared to the ones of vehicle-treated mice (Figure 6 D-E). Thus, inhibition of FPR2 signaling protected mice from IAV replication, inflammation in the lungs and severe disease development when used with a prophylactic or curative intent. 


\section{DISCUSSION}

The present study showed that the FPR2 antagonists PBP10 and BOC2 are potent antiviral molecules in vitro against a broad range of IAV and B viruses. Consistently, our previous report showed that FPR2 plays a deleterious role during IAV infections and that another FPR2 antagonist WRW4 inhibits IAV replication in vitro and in vivo. Mechanistically, the effect of PBP10 and BOC2 was abolished by treating the cells with U0126, a specific ERK pathway inhibitor. The antiviral role of these molecules occurs through ERK activation, a pathway necessary for the viral life cycle in vitro and in vivo (Droebner et al., 2011; Marjuki et al., 2011; Pleschka et al., 2001).

In vivo, administration of BOC2 to infected mice protected them from lethal IAV infections. These results confirm our previous preclinical studies showing that another inhibitor of FPR2, WRW4 efficiently protected mice against lethal IAV infections (Tcherniuk et al., 2016).

Protein sequence alignment (Figure 7) shows $76 \%$ amino acid identity between human and mouse FPR2 (85\% when considering similar residues) and 97\% amino acid identity between human and monkey FPR2 (98\% when considering similar residues). This sequence similarity is likely to explain the conserved inhibitory effect of WRW4 and BOC2 in mice.

It is noteworthy that WRW4 or BOC2 administration in a prophylactic manner also had a protective effect. Thus, FPR2 antagonism might be explored not only as a new treatment for influenza but also to prevent the disease. This effect would be particularly valuable in case of a pandemic. Indeed, although preventive vaccination exists, based on our knowledge of previous pandemic plans, the delay of 6-12 months to produce a pandemic vaccine cannot fit with a required rapid response (Webby and Webster, 2003). Vaccines are reduced to specific viral strains that should first be identified, produced in large amount and their inactivation controlled. In addition, they are accessible only to a small, privileged fraction of the world 
population. Finally, the increasing skepticism towards vaccination has led to a drop in immunization coverage rates. The development of new antiviral drugs thus appears as a relevant strategy. Regarding the advantage of FPR2 antagonists acting in a therapeutic or prophylactic manner, it would not only prevent virus from spreading from human to human but also protect the population before infection occurs. In contrast to the current antiviral drugs, FPR2 antagonists could most likely be used without the emergence of resistant viruses. Indeed, the current commercialized antivirals target viral proteins which are highly subjected to mutations. In contrast, blocking a cellular receptor will slow down viral growth and at the same time diminish the probability of the virus escaping from mutation pressure since the virus is unable to modify the host genome.

It is also noteworthy that mice lacking FPR2 develop normally, and their lifespan in a pathogen-free facility is equivalent to wild-type mice (Chen et al., 2010). This suggests that FPR2 is not a crucial receptor for cellular functions which reinforces the interest of testing FPR2 inhibitors as novel therapeutic against influenza.

It is also noteworthy that FPR2 belongs to the family of G-protein coupled receptors (GPCRs). GPCRs have been one of the most popular targets for drug developers. According to a recent publication, 30-50\% of commercialized drugs exert their effect through GPCRs and from 2005-2014, $25 \%$ of novel approved drug from US Food and Drug Administration target GPCRs (Fang et al., 2015). FPR2 plays a key role in inflammatory processes and thus is also a major target for drug developers. However, to our knowledge FPR2 inhibitors did not go through clinical trials yet. The reasons might be multiple. First, in comparison to other receptors, the precise role of FPR2 (pro-inflammatory versus resolution of inflammation) is only emerging. Then, FPR2 also belongs to the FPR family, in which two other FPRs were described in humans (FPR1 and 3) and all FPRs have similarities in their amino acid sequences. While WRW4 and PBP10 are highly specific inhibitors of FPR2, BOC2 is less 
specific and also inhibits FPR1. Thus, a limitation in the use of FPR2 antagonists is to develop very specific small molecules against FPR2. Unfortunately, the discovery of specific molecules targeting GPCRs is very intractable and currently, bio-therapeutic has been demonstrated to be a better approach (Mujic-Delic et al., 2014). Antibodies to GPCRs have been difficult to develop since they are very unstable when purified. However, with the recent technical progress made, antibodies/nanobodies directed against FPR2 will most likely be very important tools in the future for drug discovery.

Regarding IAV, 18 types of hemagglutinin and 11 types of neuraminidase were described and none of the commercialized antiviral drugs are susceptible to protect against all strains that will emerge from the animal reservoir (Webby and Webster, 2003). The strong dependencies of influenza viruses on well known specific cellular functions appear particularly relevant for the development of universal antivirals. As shown here, antagonists of FPR2 inhibited replication of several strains of influenza A and B viruses. FPR2 antagonists act through a delay in ERK activation, a signaling pathway required for endosomal acidification and viralendosomal fusion (Marjuki et al., 2011), viral ribonucleoprotein (vRNP) translocation from the nucleus to the cytoplasm and viral replication (Droebner et al., 2011; Pleschka et al., 2001). Thus, FPR2 inhibitors might impair different steps of the virus cell cycle. Also, since the acidification of the endosome and vRNP translocation is required for all strains of influenza virus life cycle, FPR2 should most likely protect against any novel influenza strain that could emerge from the animal reservoir and cause a pandemic.

Very interestingly, FPR2 antagonists showed an additive effect with oseltamivir regarding inhibition of virus replication. These results are consistent with the non-overlapping mechanism of action of both molecules. FPR2 antagonists act on the inhibition of ERK activation, while oseltamivir prevents virus release from the infected cells through inhibition 
of the viral neuraminidase. Altogether, the present paper not only confirms the deleterious role of FPR2 during IAV infections but also shows for the first time that (i) FPR2 acts through inhibition of virus replication, (ii) FPR2 antagonists used in combination with oseltamivir show additive antiviral effects, (iii) FPR2 antagonists protect mice from lethal infections induced by influenza viruses in a prophylactic manner and (iv) FPR2 antagonists inhibit influenza B virus replication. Thus, this report suggests that the use of FPR2 antagonists in combination with current antiviral drugs could be an interesting strategy to develop novel antiviral drugs.

\section{CONCLUSIONS}

FPR2 antagonist are expected to (i) show efficacy to block virus replication, (ii) limit the emergence of virus resistance, (iii) have a broad spectrum of action, regardless of the strain of influenza virus, (iv) have limited adverse effects in humans, (v) be effective when administered early or late post-infection and (vi) to boost the antiviral activity of current antiviral drugs such as oseltamivir when used in combination therapy. Altogether, this reports suggests that targeting FPR2 might offer several advantages to treat severe influenza.

\section{ACKNOWLEDGEMENTS}

This work was supported by the ANR (ANR-13-BSV3-0011), Fondation de France (Grant 00066467), Fondation Vaincre la mucoviscidose and Association Gregory Lemarchal (Grant $\left.\mathrm{N}^{\circ} \mathrm{RF} 20160501641\right)$ and SATT Sud-Est. Authors are grateful to Elodie Dormes and Florence Alesandrini for helpful advices. We thank Bekendam Roelof and Remy Collier for reading the manuscript regarding english grammar corrections. We thank A. Harmache (INRA, Nouzilly, France) for advice regarding vRNA QRT-PCR experiments. A patent has been filed on the 
role of FPR2 antagonists against influenza viruses by Beatrice Riteau and Marie-Christine Alessi. 


\section{FIGURES LEGEND}

\section{Figure 1: Cell viability and inhibition of viral genome replication by PBP10 and BOC2}

(A) A549 cells were treated with $1,25-320 \mu \mathrm{M}$ of PBP10 (white panels) or BOC2 (grey panels) and cell viability was estimated by trypan blue staining 72 hours onwards. (B) A549 cells were pretreated with $5 \mu \mathrm{M}$ of PBP10 (left panel) or BOC2 (right panel) and infected with IAV A/PR/8/34 virus at a MOI of 1 . At the indicated time points after infection, infectious virus titers were determined by plaque assay. (C) A549 cells were pretreated with different concentrations of PBP10 (left panel) or BOC2 (right panel) and infected with $\mathrm{A} / \mathrm{PR} / 8 / 34$ virus at a MOI of 1 . Twenty-four hours post-infection, virus titers were determined by plaque assay. NI: Non infected; I: Infected. All experiments are representative of at least two independent assays.

\section{Figure 2: Antiviral activity of PBP10 and BOC2}

(A) A549 cells were treated or not with PBP10 or BOC2 $(5 \mu \mathrm{M})$ and infected with IAV A/PR/8/34 virus (MOI 1). At 24 hours post-infection, M2 protein synthesis was visualized by immunofluorescence microscopy, using an anti-M2 antibody. The nuclei were stained with DAPI and the merged images are shown. (B) A549 cells were pre-incubated with $5 \mu \mathrm{M}$ of either BOC2 or PBP10 (or vehicle) and then infected with IAV A/PR/8/34 virus (MOI 1). RNA was extracted and real-time qPCRs were performed with specific primers to quantify gene expression of viral NS1 protein (mRNA or vRNA) at the indicated time point postinfection. Data are represented as means \pm SEM, $n=3-6$ replicates. 


\section{Figure 3: PBP10 and BOC2 inhibit IAV-induced ERK activation}

(A-B) A549 cells were incubated with A/PR/8/34 viruses (MOI 10) for the indicated time point (minutes) in presence or absence of PBP10 (A) or BOC2 (B). Cells were then lysed and ERK phosphorylation was analysed by western blotting. (C) A549 cells were first preincubated or not with PBP10 or BOC2 and then infected with A/PR/8/34 virus in presence or absence of U0126. Infectious virus titers were determined by plaque assay 24 hours postinfection.

\section{Figure 4: Co-treatment of FPR2 antagonists plus oseltamivir}

The antiviral effect of FPR2 antagonist alone, oseltamivir alone or a combination of FPR2 antagonist and oseltamivir was evaluated on A/PR/8/34-infected A549 cells. Infectious virus titers were determined by plaque assay 24 hours post-infection.

\section{Figure 5: Antiviral activity of FPR2 antagonists is independent on virus strain}

A549 cells were first pretreated or not with $5 \mu \mathrm{M}$ of FPR2 antagonists PBP10, BOC2 or WRW4. Cells were then infected with influenza virus A/HK/68 (IAV, H3N2), A/Turkey/Massachusetts/65 (IAV, H6N2) or B/NL/076/06 and 24 hours post-infection, infectious virus titers were determined by plaque assay.

\section{Figure 6: FPR2 antagonists protect from influenza virus pathogenesis}

(A) Survival of mice treated with BOC2 or vehicle ( $n=8 /$ group) and infected the same day with IAV A/PR/8/34 (500 PFU/mouse). (B) Survival of mice treated with BOC2 or vehicle ( $\mathrm{n}=5 /$ group) and infected the next day with IAV A/PR/8/34 (750 PFU/mouse). (C) Survival of mice treated with WRW4 or vehicle ( $n=5 /$ group) and infected the next day with IAV A/PR/8/34 (750 PFU/mouse). (D, E) Mice were treated with WRW4 or vehicle ( $n=5 /$ group) 
and then subsequently infected on the next day with IAV A/PR/8/34 (750 PFU/mouse). Infectious lung virus titers (D) or total proteins in the BAL of vehicle or WRW4-treated mice were evaluated at day 6 post-infection. Data represent mean \pm s.e.m of 3 individual mice per group.

\section{Figure 7: Sequence alignment of FPR2}

The sequences of FPR2 from mouse and monkey were compared to the one of human FPR2. The percentage identity represents residues that are identical. Positives are residues that are very similar to each other. 


\section{REFERENCES}

Bae, Y.S., Lee, H.Y., Jo, E.J., Kim, J.I., Kang, H.K., Ye, R.D., Kwak, J.Y., Ryu, S.H., 2004. Identification of peptides that antagonize formyl peptide receptor-like 1-mediated signaling. $\mathbf{J}$ Immunol 173, 607-614.

Baier, G., Telford, D., Gulbins, E., Yamada, N., Kawakami, T., Altman, A., 1993. Improved specificity of RT-PCR amplifications using nested cDNA primers. Nucleic Acids Res 21, 1329-1330.

Berri, F., Haffar, G., Le, V.B., Sadewasser, A., Paki, K., Lina, B., Wolff, T., Riteau, B., 2014. Annexin $\mathrm{V}$ incorporated into influenza virus particles inhibits gamma interferon signaling and promotes viral replication. J Virol 88, 11215-11228.

Berri, F., Rimmelzwaan, G.F., Hanss, M., Albina, E., Foucault-Grunenwald, M.L., Le, V.B., Vogelzang-van Trierum, S.E., Gil, P., Camerer, E., Martinez, D., Lina, B., Lijnen, R., Carmeliet, P., Riteau, B., 2013. Plasminogen controls inflammation and pathogenesis of influenza virus infections via fibrinolysis. PLoS Pathog 9, e1003229.

Chen, K., Le, Y., Liu, Y., Gong, W., Ying, G., Huang, J., Yoshimura, T., Tessarollo, L., Wang, J.M., 2010. A critical role for the g protein-coupled receptor mFPR2 in airway inflammation and immune responses. J Immunol 184, 3331-3335.

Cunningham, C.C., Vegners, R., Bucki, R., Funaki, M., Korde, N., Hartwig, J.H., Stossel, T.P., Janmey, P.A., 2001. Cell permeant polyphosphoinositide-binding peptides that block cell motility and actin assembly. J Biol Chem 276, 43390-43399.

Deng, X., Ueda, H., Su, S.B., Gong, W., Dunlop, N.M., Gao, J.L., Murphy, P.M., Wang, J.M., 1999. A synthetic peptide derived from human immunodeficiency virus type 1 gp120 downregulates the expression and function of chemokine receptors CCR5 and CXCR4 in 
monocytes by activating the 7-transmembrane G-protein-coupled receptor FPRL1/LXA4R. Blood 94, 1165-1173.

Droebner, K., Pleschka, S., Ludwig, S., Planz, O., 2011. Antiviral activity of the MEKinhibitor U0126 against pandemic H1N1v and highly pathogenic avian influenza virus in vitro and in vivo. Antiviral Res 92, 195-203.

Fang, Y., Kenakin, T., Liu, C., 2015. Editorial: Orphan GPCRs As Emerging Drug Targets. Front Pharmacol 6, 295.

Fu, H., Bjorkman, L., Janmey, P., Karlsson, A., Karlsson, J., Movitz, C., Dahlgren, C., 2004. The two neutrophil members of the formylpeptide receptor family activate the NADPHoxidase through signals that differ in sensitivity to a gelsolin derived phosphoinositidebinding peptide. BMC Cell Biol 5, 50.

Hayden, F.G., de Jong, M.D., 2011. Emerging influenza antiviral resistance threats. J Infect Dis 203, 6-10.

Hoffmann, E., Stech, J., Guan, Y., Webster, R.G., Perez, D.R., 2001. Universal primer set for the full-length amplification of all influenza A viruses. Arch Virol 146, 2275-2289.

Kuiken, T., Riteau, B., Fouchier, R.A., Rimmelzwaan, G.F., 2012. Pathogenesis of influenza virus infections: the good, the bad and the ugly. Curr Opin Virol 2, 276-286.

Le, V.B., Schneider, J.G., Boergeling, Y., Berri, F., Ducatez, M., Guerin, J.L., Adrian, I., Errazuriz-Cerda, E., Frasquilho, S., Antunes, L., Lina, B., Bordet, J.C., Jandrot-Perrus, M., Ludwig, S., Riteau, B., 2015. Platelet activation and aggregation promote lung inflammation and influenza virus pathogenesis. Am J Respir Crit Care Med 191, 804-819.

Le, Y., Murphy, P.M., Wang, J.M., 2002. Formyl-peptide receptors revisited. Trends in immunology 23, 541-548. 
Lin, Q., Fang, D., Hou, X., Le, Y., Fang, J., Wen, F., Gong, W., Chen, K., Wang, J.M., Su, S.B., 2011. HCV peptide (C5A), an amphipathic alpha-helical peptide of hepatitis virus C, is an activator of $\mathrm{N}$-formyl peptide receptor in human phagocytes. J Immunol 186, 2087-2094. Marjuki, H., Gornitzky, A., Marathe, B.M., Ilyushina, N.A., Aldridge, J.R., Desai, G., Webby, R.J., Webster, R.G., 2011. Influenza A virus-induced early activation of ERK and PI3K mediates V-ATPase-dependent intracellular $\mathrm{pH}$ change required for fusion. Cell Microbiol 13, $587-601$.

Mujic-Delic, A., de Wit, R.H., Verkaar, F., Smit, M.J., 2014. GPCR-targeting nanobodies: attractive research tools, diagnostics, and therapeutics. Trends Pharmacol Sci 35, 247-255.

Nedellec, R., Coetzer, M., Shimizu, N., Hoshino, H., Polonis, V.R., Morris, L., Martensson, U.E., Binley, J., Overbaugh, J., Mosier, D.E., 2009. Virus entry via the alternative coreceptors CCR3 and FPRL1 differs by human immunodeficiency virus type 1 subtype. J Virol 83, 8353-8363.

Ortiz-Munoz, G., Mallavia, B., Bins, A., Headley, M., Krummel, M.F., Looney, M.R., 2014. Aspirin-triggered 15-epi-lipoxin A4 regulates neutrophil-platelet aggregation and attenuates acute lung injury in mice. Blood 124, 2625-2634.

Pleschka, S., Wolff, T., Ehrhardt, C., Hobom, G., Planz, O., Rapp, U.R., Ludwig, S., 2001. Influenza virus propagation is impaired by inhibition of the Raf/MEK/ERK signalling cascade. Nature cell biology 3, 301-305.

Riteau, B., Barber, D.F., Long, E.O., 2003. Vav1 phosphorylation is induced by beta2 integrin engagement on natural killer cells upstream of actin cytoskeleton and lipid raft reorganization. J Exp Med 198, 469-474.

Shimizu, N., Tanaka, A., Mori, T., Ohtsuki, T., Hoque, A., Jinno-Oue, A., Apichartpiyakul, C., Kusagawa, S., Takebe, Y., Hoshino, H., 2008a. A formylpeptide receptor, FPRL1, acts as 
an efficient coreceptor for primary isolates of human immunodeficiency virus. Retrovirology 5,52 .

Shimizu, N., Tanaka, A., Oue, A., Mori, T., Apichartpiyakul, C., Hoshino, H., 2008b. A short amino acid sequence containing tyrosine in the N-terminal region of $\mathrm{G}$ protein-coupled receptors is critical for their potential use as co-receptors for human and simian immunodeficiency viruses. J Gen Virol 89, 3126-3136.

Skovbakke, S.L., Heegaard, P.M., Larsen, C.J., Franzyk, H., Forsman, H., Dahlgren, C., 2015. The proteolytically stable peptidomimetic Pam-(Lys-betaNSpe)6-NH2 selectively inhibits human neutrophil activation via formyl peptide receptor 2. Biochemical pharmacology 93, 182-195.

Su, S.B., Gao, J., Gong, W., Dunlop, N.M., Murphy, P.M., Oppenheim, J.J., Wang, J.M., 1999. T21/DP107, A synthetic leucine zipper-like domain of the HIV-1 envelope gp41, attracts and activates human phagocytes by using G-protein-coupled formyl peptide receptors. J Immunol 162, 5924-5930.

Tcherniuk, S., Cenac, N., Comte, M., Frouard, J., Errazuriz-Cerda, E., Galabov, A., Morange, P.E., Vergnolle, N., Si-Tahar, M., Alessi, M.C., Riteau, B., 2016. Formyl Peptide Receptor 2 Plays a Deleterious Role During Influenza A Virus Infections. J Infect Dis 214, 237-247.

Verriere, V., Higgins, G., Al-Alawi, M., Costello, R.W., McNally, P., Chiron, R., Harvey, B.J., Urbach, V., 2012. Lipoxin A4 stimulates calcium-activated chloride currents and increases airway surface liquid height in normal and cystic fibrosis airway epithelia. PLoS One 7, e37746.

Vital, S.A., Becker, F., Holloway, P.M., Russell, J., Perretti, M., Granger, D.N., Gavins, F.N., 2016. Formyl-Peptide Receptor 2/3/Lipoxin A4 Receptor Regulates Neutrophil-Platelet Aggregation and Attenuates Cerebral Inflammation: Impact for Therapy in Cardiovascular Disease. Circulation 133, 2169-2179. 
Webby, R.J., Webster, R.G., 2003. Are we ready for pandemic influenza? Science 302, 15191522. 